\title{
Real Time Temperature Graph using MATLAB and Arduino
}

\author{
Ritwik Biswas \\ B Tech,Dept of ECE \\ JIS College of Engineering,Kalyani INDIA
}

\author{
Avijit Roy \\ B Tech,Dept of ECE \\ JIS College of Engineering, Kalyani INDIA
}

\begin{abstract}
This project illustrates the use of MATLAB along with ARDUINO for monitoring the temperature of the surroundings. The ARDUINO platform has become so versatile that it has developed many software to support several different applications on it, it also has developed software to support MATLAB, which allows to obtain and plot Real Time data directly from within the MATLAB command window from Arduino. This real time data can be used to observe the temperature and change it according to our needs.
\end{abstract}

\section{INTRODUCTION}

A temperature sensor is a device, typically, a thermocouple or RTD that provides for temperature measurement through an electrical signal. A thermocouple (T/C) is made from two dissimilar metals that generate electrical voltage in direct proportion to change in temperature. An RTD (Resistance Temperature Detector) is a variable resistor that will change its electrical resistance in direct proportion to changes in temperature in a precise, repeatable and nearly linear manner.

Temperature Sensors measure the amount of heat energy or even coldness that is generated by an object or system, allowing us to "sense" or detect any physical change to that temperature producing either an analogue or digital output.

The LM35 series are precision integrated-circuit temperature devices with an output voltage linearly proportional to the Centigrade temperature. The LM35 device has an advantage over linear temperature sensors calibrated in Kelvin, as the user is not required to subtract a large constant voltage from the output to obtain convenient Centigrade scaling. The LM35 device does not require any external calibration or trimming to provide typical accuracies of $\pm 1 / 4{ }^{\circ} \mathrm{C}$ at room temperature and $\pm 3 / 4{ }^{\circ} \mathrm{C}$ over a full $-55^{\circ} \mathrm{C}$ to $150^{\circ} \mathrm{C}$ temperature range. Lower cost is assured by trimming and calibration at the wafer level. The low-output impedance, linear output and precise inherent calibration of the LM35 device makes interfacing to readout or control circuitry especially easy.

They are more sensitive than other temperature sensors. High sensitivity allows them to work well over a small temperature range. They're low cost and therefore cheap to replace. They provide a fast response.

\section{PRINCIPLE OF WORKING OF TEMPERATURE SENSOR}

We are intended to store data from a temperature sensor in an Arduino Uno board and displaying it on the Matlab GUI while also plotting the data on a graph in real time to monitor sensor changes.

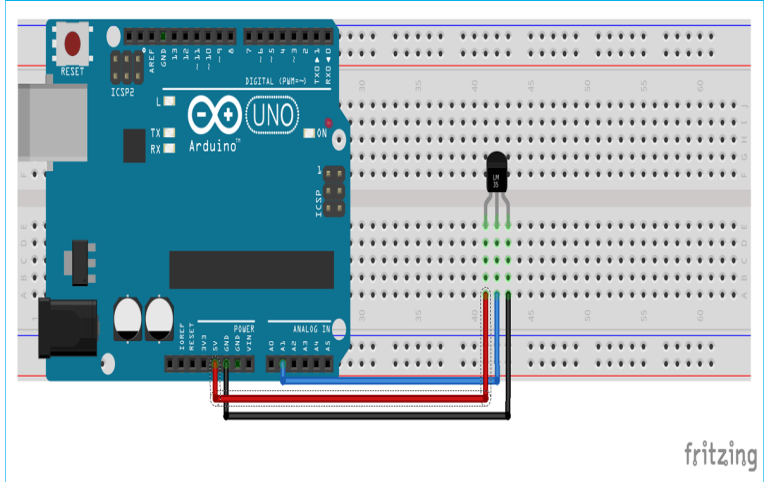

The temperature sensor which we are going to use in this project is LM35, which is connected to the Arduino with the help of breadboard and the jumper wires.

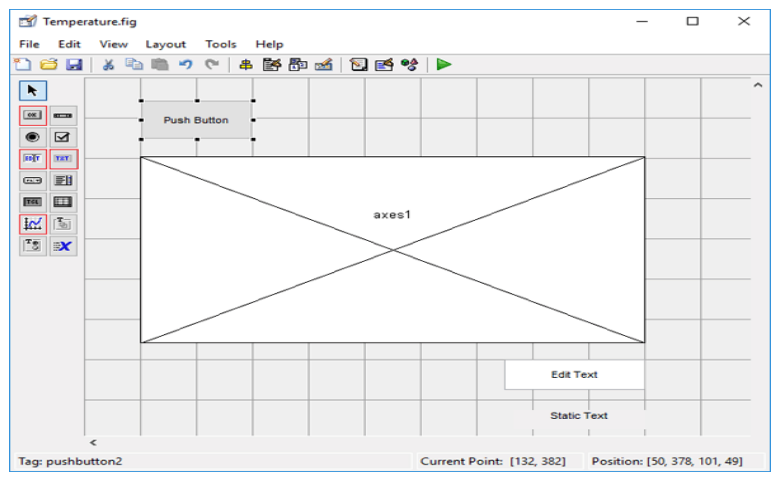

The main part of the project is the GUI section of the Matlab. With the help of this gui we have a created an application where a start button is given which is used to start the reading recording.

The axes is given which is used to plot the temperature The $\mathrm{x}$ axis indicates time

The y axis indicates temperature. 


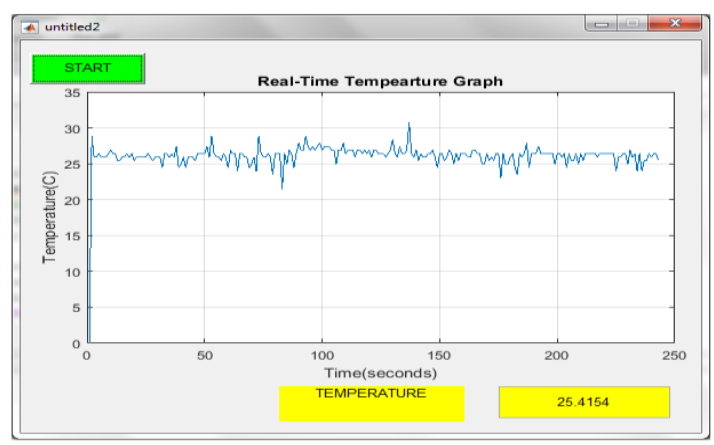

LM35 outputs a voltage with respect to change in temperature, this output is measured using Arduino analog to digital converter, and then converted into temperature value, which is then displayed on the Matlab command window and then with the help of GUI the readings are plotted in the graph.

As I am using MATLAB for this project, that's why to send data from Arduino to MATLAB, we have used the Arduino Matlab support package, by using this package we can create two virtual communication ports and connect them, after doing this, whatever we send on first port will get received on second port and vice versa.

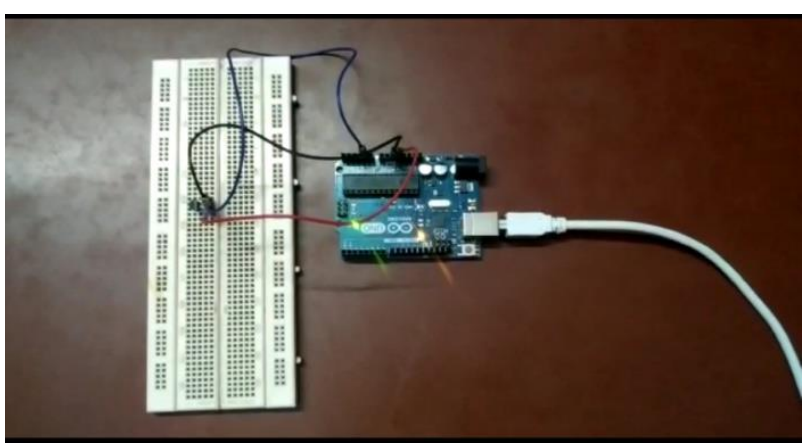

This is our final project with the Arduino in use. As the LM35 temperature sensor senses the temperature, it is then recorded by the Arduino and at last is displayed on the Matlab command window.

By adding a resistor to the above project i.e. connecting it with the LM35 temperature sensor, we can increase the temperature range of the LM35. So that it can give us a wider range of temperature to access the temperature conditions precisely.

It can also act as a protective gear for our project. For any uneven fluctuations or short-circuits the resistor can resist it.

\section{CONCLUSION}

The project was used to determine the temperature of a place or surrounding using the LM35 temperature sensor. It was connected with the Arduino and the outputs were taken in MATLAB. With the help of MATLAB we were able to create a temperature graph and the outputs were recorded accordingly. An interface was created with the help of MATLAB GUI to control the functioning of the graph. In the future we look forward to make this temperature sensor into a large scale project by adding additional features so that it can be very useful in the modern day society.

\section{REFERENCES}

[1] https://www.electronicwings.com/components/lm35temperature-sensor

[2] https://randomnerdtutorials.com/arduino- $\operatorname{lm} 35-\operatorname{lm} 335-\operatorname{lm} 34-$ temperature-sensor/

[3] MATLAB GUI Tutorial for Beginners - YouTube

[4] How to Plot Real Time Temperature Graph using MATLAB YouTube

[5] https://www.instructables.com/id/ARDUINO-TEMPERATURESENSOR-LM35/ 